\title{
PUBLIC KEY EXCHANGE USING MATRICES OVER GROUP RINGS
}

\author{
DELARAM KAHROBAEI, CHARALAMBOS KOUPPARIS, AND VLADIMIR SHPILRAIN
}

\begin{abstract}
We offer a public key exchange protocol in the spirit of Diffie-Hellman, but we use (small) matrices over a group ring of a (small) symmetric group as the platform. This "nested structure" of the platform makes computation very efficient for legitimate parties. We discuss security of this scheme by addressing the Decision Diffie-Hellman (DDH) and Computational Diffie-Hellman ( $\mathrm{CDH})$ problems for our platform.
\end{abstract}

\section{INTRODUCTION}

The beginning of public key cryptography can be traced back to the paper by Diffie and Hellman [2]. The simplest, and original, implementation of their key exchange protocol uses $\mathbb{Z}_{p}^{*}$, the multiplicative group of integers modulo a prime $p$, as the platform. There is also a public element $g \in \mathbb{Z}_{p}$, which is a primitive root $\bmod p$. The protocol itself is as follows:

(1) Alice chooses an integer $a$, computes $A=g^{a} \bmod p$ and publishes $A$

(2) Bob picks an integer $b$ and computes $B=g^{b} \bmod p$, and publishes $B$

(3) Alice computes $K_{A}=B^{a} \bmod p$

(4) Bob computes $K_{B}=A^{b} \bmod p$

Both Alice and Bob are now in possession of a secret shared key $K$, as $g^{a b} \bmod p=$ $g^{b a} \bmod p$ and hence $K:=K_{A}=K_{B}$.

The protocol is considered secure provided $G$ and $g$ are chosen properly, see e.g. [5] for details. In order to recover the shared secret key, the eavesdropper Eve must be able to solve the Diffie-Hellman problem (recover $g^{a b}$ from $g, g^{a}$ and $g^{b}$ ). One could solve the DiffieHellman problem by solving the discrete logarithm problem, i.e., by recovering $a$ from $g$ and $g^{a}$. However, it is unknown whether the discrete logarithm problem is equivalent to the Diffie-Hellman problem.

We should note that there is still the "brute force" method of solving the discrete logarithm problem. The eavesdropper can simply start computing successively higher powers of $g$, until they match $g^{a}$. This requires at most $|g|$ multiplications, where $|g|$ is the order of $g$ in the group $G$. It is usually the case however that $|g| \approx 10^{300}$ and hence this method is considered computationally infeasible.

Initially it may seem that the legitimate parties, Alice and Bob, will also have to perform a large number of multiplications, thus facing the same problem as the eavesdropper does. However, as the legitimate parties are in possession of $a$ and $b$, they can use the "square and multiply" algorithm that requires $O\left(\log _{2} a\right)$ multiplications, e.g. $g^{27}=\left(\left(\left(g^{2}\right)^{2}\right)^{2}\right)^{2} \cdot\left(\left(g^{2}\right)^{2}\right)^{2}$. $g^{2} \cdot g$.

Research of the first author was partially supported by a PSC-CUNY grant from the CUNY research foundation, as well as the City Tech foundation.

Research of the third author was partially supported by the NSF grants DMS-0914778 and CNS-1117675. 
There is some disadvantage to working with $\mathbb{Z}_{p}$, where $p, a$, and $b$ are chosen to be fairly large. Computation with 300-digit numbers (or 1000-bit binary numbers) is not particularly efficient, and neither is reducing the result modulo $p$. This is one of the reasons why the DiffieHellman key agreement protocol with recommended parameters is not suitable for devices with limited computational resources. Hence, there is an ongoing search for other platforms where the Diffie-Hellman or a similar key exchange can be carried out more efficiently, in particular with public and/or private keys of smaller size.

The platform that we are proposing here is the semigroup of matrices (of a small size) over a group ring, with the usual matrix multiplication operation. More specifically, we are working with matrices over the group ring $\mathbb{Z}_{n}\left[S_{m}\right]$, where $\mathbb{Z}_{n}$ is the ring of integers modulo $n$ and $S_{m}$ is the symmetric group of degree $m$. To verify the security of using such a semigroup of matrices as the platform, we address the Computational Diffie-Hellman and Decision Diffie-Hellman problems (Section 3), along with questions about the structure of this semigroup.

Parameters that we suggest $\left(2 \times 2\right.$ or $3 \times 3$ matrices over $\left.\mathbb{Z}_{7}\left[S_{5}\right]\right)$ provide for a large key space $\left(7^{480} \sim 10^{406}\right.$ for $2 \times 2$ matrices and $7^{1080} \sim 10^{913}$ for $3 \times 3$ matrices $)$. Storing a single $2 \times 2$ matrix over $\mathbb{Z}_{7}\left[S_{5}\right]$ takes about 1440 bits, and a single $3 \times 3$ matrix about 3240 bits, so keys are of about the same size as in the "classical" Diffie-Hellman scheme (storing an integer

of size about $10^{300}$ requires 997 bits). These storage requirements can be reduced by $\frac{1}{7} t h$ if we do not store polynomial terms which have a 0 as their coefficient, thus bringing the key size down to about 1230 bits for $2 \times 2$ matrices and to about 2780 bits for $3 \times 3$ matrices.

What we believe is one of the main advantages of our platform over the standard $\mathbb{Z}_{p}$ platform in the original Diffie-Hellman scheme is that the multiplication of matrices over $\mathbb{Z}_{7}\left[S_{5}\right]$ is very efficient. In particular, in our setup multiplying elements is faster than multiplying numbers in $\mathbb{Z}_{p}$ for a large $p$. This is due to the fact that one can pre-compute the multiplication table for the group $S_{5}$ (of order 120), so in order to multiply two elements of $\mathbb{Z}_{7}\left[S_{5}\right]$ there is no "actual" multiplication in $S_{5}$ involved, but just re-arranging a bit string and multiplying coefficients in $\mathbb{Z}_{7}$. Also, in our multiplication there is no reduction of the result modulo $p$ that slows down computation in $\mathbb{Z}_{p}$ for a large $p$. Informally speaking, the "nested structure" of our platform (small matrices over a group ring of a small group $S_{5}$ over a small ring $\mathbb{Z}_{7}$ ) provide for more efficient computation than just using $\mathbb{Z}_{p}$ with a very large $p$.

From a security standpoint, an advantage of our platform over the group $\mathbb{Z}_{p}$, or elliptic curves, is that "standard" attacks (baby-step giant-step, Pohlig-Hellman, Pollard's rho) do not work with our platform, as we show in Section 6. Furthermore, our platform proves secure against Shor's quantum algorithm which is a common pitfall on classical Diffie-Hellman algorithms, see Section 6.3 .

\section{Group Rings}

Definition 2.1. Let $G$ be a group written multiplicatively and let $R$ be any commutative ring with nonzero unity. The group ring $R[G]$ is defined to be the set of all formal sums

$$
\sum_{g_{i} \in G} r_{i} g_{i}
$$

where $r_{i} \in R$, and all but a finite number of $r_{i}$ are zero.

We define the sum of two elements in $R[G]$ by 


$$
\left(\sum_{g_{i} \in G} a_{i} g_{i}\right)+\left(\sum_{g_{i} \in G} b_{i} g_{i}\right)=\sum_{g_{i} \in G}\left(a_{i}+b_{i}\right) g_{i} .
$$

Note that $\left(a_{i}+b_{i}\right)=0$ for all but a finite number of $i$, hence the above sum is in $R[G]$. Thus $(R[G],+)$ is an abelian group.

Multiplication of two elements of $R[G]$ is defined by the use of the multiplications in $G$ and $R$ as follows:

$$
\left(\sum_{g_{i} \in G} a_{i} g_{i}\right)\left(\sum_{g_{i} \in G} b_{i} g_{i}\right)=\sum_{g_{i} \in G}\left(\sum_{g_{j} g_{k}=g_{i}} a_{j} b_{k}\right) g_{i} .
$$

As an example of a group ring, we consider the symmetric group $S_{5}$ and the ring $\mathbb{Z}_{7}$ and form the group ring $\mathbb{Z}_{7}\left[S_{5}\right]$. We will write the identity element of $S_{m}$ as $e$. Sample elements and operations are

$$
\begin{aligned}
a & =5(123)+2(15)(24)+(153) \\
b & =3(123)+4(1453) \\
a+b & =(123)+2(15)(24)+(153)+4(1453) \\
a b & =(5(123)+2(15)(24)+(153))(3(123)+4(1453)) \\
& =15(132)+20(145)(23)+6(14235)+8(124)(35)+3(12)(35)+4(1435) \\
& =(132)+6(145)(23)+6(14235)+(124)(35)+3(12)(35)+4(1435) \\
b a & =(3(123)+4(1453))(5(123)+2(15)(24)+(153)) \\
& =15(132)+6(15243)+3(15)(23)+20(12)(345)+8(13)(254)+4(1345) \\
& =(132)+6(15243)+3(15)(23)+6(12)(345)+(13)(254)+4(1345)
\end{aligned}
$$

Now that group rings have been defined, it is clear how to define $M_{2}\left(\mathbb{Z}_{n}\left[S_{m}\right]\right)$, the ring of $2 \times 2$ matrices over the group ring $\mathbb{Z}_{n}\left[S_{m}\right]$. We are only going to be concerned with multiplication of matrices in this ring; as an example using the same $a$ and $b$ defined above, we can define

$$
M_{1}=\left[\begin{array}{ll}
a & e \\
e & b
\end{array}\right], M_{2}=\left[\begin{array}{ll}
b & e \\
0 & a
\end{array}\right] .
$$

Then

$$
\begin{aligned}
M_{1} M_{2} & =\left[\begin{array}{cc}
a b & 2 a \\
b & e+b a
\end{array}\right] \\
& =\left[\begin{array}{cc}
a b & 3(123)+4(15)(24)+2(153) \\
3(123)+4(1453) & e+b a
\end{array}\right],
\end{aligned}
$$

where $a b$ and $b a$ are computed above.

\section{Computational Diffie-Hellman and Decision Diffie-Hellman}

Recall that in the Diffie-Hellman key exchange Alice and Bob want to establish a secret shared key. Alice chooses a finite group $G$ and an element $g$ of the group $G$. Alice then picks a random $a$ and publishes $\left(g, G, g^{a}\right)$. Bob also picks a random $b$ and publishes $\left(g^{b}\right)$. 
Alice's and Bob's secret key is now $g^{a b}$, which can be computed by both of them since $g^{a b}=\left(g^{a}\right)^{b}=\left(g^{b}\right)^{a}$. The security of the Diffie-Hellman key exchange relies on the assumption that it is computationally hard to recover $g^{a b}$ given $\left(g, G, g^{a}, g^{b}\right)$.

A passive eavesdropper, Eve, would try to recover $g^{a b}$ from $\left(g, G, g^{a}, g^{b}\right)$. One defines the Diffie-Hellman algorithm by $F\left(g, G, g^{a}, g^{b}\right)=g^{a b}$. We say that a group $G$ satisfies the Computational Diffie-Hellman (CDH) assumption if no efficient algorithm exists to compute $F\left(g, G, g^{a}, g^{b}\right)=g^{a b}$. More precisely,

Definition 3.1. A CDH algorithm $F$ for a group $G$ is a probabilistic polynomial time algorithm satisfying, for some fixed $\alpha>0$ and all sufficiently large $n$,

$$
\mathbb{P}\left[F\left(g, G, g^{a}, g^{b}\right)=g^{a b}\right]>\frac{1}{n^{\alpha}} .
$$

The probability is over a uniformly random choice of a and $b$. We say that the group $G$ satisfies the CDH assumption if there is no $C D H$ algorithm for $G$.

Even though a group may satisfy the $\mathrm{CDH}$ assumption, $\mathrm{CDH}$ by itself is not sufficient to prove that the Diffie-Hellman protocol is useful for practical cryptographic purposes. While Eve may not be able to recover the entire secret, she may still be able to recover valuable information about it. For example, even if $\mathrm{CDH}$ is true, Eve may still be able to predict $80 \%$ of the bits of $g^{a b}$ with reasonable confidence [1].

Hence if we are using $g^{a b}$ as the shared secret key, one must be able to bound the information Eve can extract about it given $g, g^{a}$ and $g^{b}$. This is formally expressed by the much stronger Decision Diffie-Hellman (DDH) assumption.

Definition 3.2. A DDH algorithm $F$ for a group $G$ is a probabilistic polynomial time algorithm satisfying, for some fixed $\alpha>0$ and all sufficiently large $n$,

$$
\mid \mathbb{P}\left[F\left(g, G, g^{a}, g^{b}, g^{a b}\right)=\text { "True" }\right]-\mathbb{P}\left[F\left(g, G, g^{a}, g^{b}, g^{c}\right)=\text { "True" }\right] \mid>\frac{1}{n^{\alpha}} .
$$

The probability is over a uniformly random choice of $a, b$ and $c$. We say that the group $G$ satisfies the DDH assumption if there is no DDH algorithm for $G$.

Essentially, the DDH assumption implies that there is no efficient algorithm which can distinguish between the two probability distributions $\left(g^{a}, g^{b}, g^{a b}\right)$ and $\left(g^{a}, g^{b}, g^{c}\right)$, where $a, b$ and $c$ are chosen at random.

\section{Diffie-Hellman key exchange protocol using matrices over $\mathbb{Z}_{n}\left[S_{m}\right]$}

While $S_{m}$ is a relatively small group for small $m$, the size of the group ring $\mathbb{Z}_{n}\left[S_{m}\right]$ grows reasonably fast, even for small values of $n$ and $m$. This is one reason we chose to look at the Diffie-Hellman key exchange protocol using these group rings. We propose to work with the group ring $\mathbb{Z}_{7}\left[S_{5}\right]$, which has the size $7^{5 !}=7^{120}$. The next step is to work with matrices over these group rings. Hence, say, the semigroup $M_{3}\left(\mathbb{Z}_{7}\left[S_{5}\right]\right)$ of $3 \times 3$ matrices has the order $\left(7^{5 !}\right)^{9} \approx 10^{913}$. This semigroup of matrices can now serve as the platform for the DiffieHellman key exchange protocol. The procedure Alice and Bob carry out is essentially the same.

Alice chooses a public matrix $M \in M_{3}\left(\mathbb{Z}_{7}\left[S_{5}\right]\right)$ and a private large positive integer $a$, computes $M^{a}$, and publishes $\left(M, M^{a}\right)$. Bob chooses another large integer $b$, and computes 
and publishes $\left(M^{b}\right)$. Both Alice and Bob can now compute the same shared secret key $K=\left(M^{a}\right)^{b}=\left(M^{b}\right)^{a}$.

As we have already mentioned in the Introduction, multiplication of matrices in the semigroup $M_{3}\left(\mathbb{Z}_{7}\left[S_{5}\right]\right)$ is very efficient, and, of course, in this semigroup, as in any other semigroup, we can use the "square and multiply algorithm" for exponentiation.

To assess security of our proposal, we should address the two Diffie-Hellman assumptions, $\mathrm{CDH}$ and DDH. We investigate the (stronger) DDH assumption experimentally in Section 5.

Finally, some of the algebraic properties of $M_{3}\left(\mathbb{Z}_{7}\left[S_{5}\right]\right)$ will be investigated.

\section{EXPERIMENTAL RESUlts}

The CDH assumption can only be answered theoretically, but the DDH assumption can be investigated experimentally. To construct our matrix semigroups we implemented the necessary group ring procedures in $\mathrm{C}++$. We have the choice of which symmetric group to use and which ring $\mathbb{Z}_{n}$ to use as well. Next we used a standard uniform distribution implementation to allow for a random selection of an element from our group ring. Finally, we constructed random $k \times k$ matrices over our group ring. Experiments were carried out with various group rings $M_{k}\left(\mathbb{Z}_{n}\left[S_{m}\right]\right)$.

We propose the use of $S_{5}$ as the group for our experiments since its underlying structure is understood and simple. When constructing the semigroup $\mathbb{Z}_{n}\left[S_{5}\right]$, one has the benefits of using the group $S_{5}$ as a building block. Namely, the group $S_{5}$ has the advantage of having only one normal subgroup, $A_{5}$, which has index 2 in $S_{5}$. Hence, trying to get some information about $a$ from $M^{a}$ by applying a non-trivial group homomorphism is limited only to the sign homomorphism $S_{5}$ to $\mathbb{Z}_{2}$ of a symmetric group.

We naturally implemented a "square and multiply" routine to speed up computations for exponentiation. With this procedure we can compute high powers of random matrices from our matrix semigroups fairly quickly, see Table 1 .

We note that the computations were carried out on an Intel Core2 Duo 2.26GHz machine, utilizing only one core, with $4 \mathrm{~GB}$ of memory and the times were computed as an average time after 250 such exponentiations. No optimizations were in effect and only one processor was used. Thus computational time may be reduced significantly by using more than one core and by implementing any available optimizations for DH using our scheme.

As a comparison for computational times, we refer to recent results of [4] claiming new speed records for DH implementations. In the paper, an implementation of the DH signature exchange protocol over the elliptic curve P-224 is presented. Without any optimization they can carry out 1800 operations per second for the DH protocol, on a somewhat more powerful computer than ours. Recall that in P-224 you require approximately 340 operations for a single "exponentiation". Hence, they require about 0.2 seconds per DH exponentiation versus our 0.6 seconds in $M_{2}\left(\mathbb{Z}_{7}\left[S_{5}\right]\right)$.

One additional thing we noticed was that the speed of computation is independent of the number of nonzero terms in the entries of our matrices $M$. One possible intuitive explanation is based on the fact that any symmetric group can be generated by a set of 2 particular elements. Since we selected 9 (or 4) random group ring elements for each matrix, there is a high probability that we have selected a pair of group elements that will generate all of 
TABle 1. Speed of Computation

\begin{tabular}{c|c|c|r} 
Matrix Size & $\mathbb{Z}_{n}$ & Exponent & Avg. Time $(\mathrm{s})$ \\
\hline $2 \times 2$ & 2 & $10^{10}$ & 0.06 \\
$2 \times 2$ & 3 & $10^{10}$ & 0.06 \\
$2 \times 2$ & 5 & $10^{10}$ & 0.06 \\
$2 \times 2$ & 7 & $10^{10}$ & 0.06 \\
\hline $2 \times 2$ & 2 & $10^{100}$ & 0.58 \\
$2 \times 2$ & 3 & $10^{100}$ & 0.58 \\
$2 \times 2$ & 5 & $10^{100}$ & 0.58 \\
$2 \times 2$ & 7 & $10^{100}$ & 0.59 \\
\hline $2 \times 2$ & 2 & $10^{1000}$ & 5.97 \\
$2 \times 2$ & 3 & $10^{1000}$ & 6.11 \\
$2 \times 2$ & 5 & $10^{1000}$ & 5.98 \\
$2 \times 2$ & 7 & $10^{1000}$ & 6.66 \\
\hline $3 \times 3$ & 2 & $10^{10}$ & 0.19 \\
$3 \times 3$ & 3 & $10^{10}$ & 0.20 \\
$3 \times 3$ & 5 & $10^{10}$ & 0.20 \\
$3 \times 3$ & 7 & $10^{10}$ & 0.20 \\
\hline $3 \times 3$ & 2 & $10^{100}$ & 1.95 \\
$3 \times 3$ & 3 & $10^{100}$ & 1.95 \\
$3 \times 3$ & 5 & $10^{100}$ & 1.94 \\
$3 \times 3$ & 7 & $10^{100}$ & 1.94 \\
\hline $3 \times 3$ & 2 & $10^{1000}$ & 20.17 \\
$3 \times 3$ & 3 & $10^{1000}$ & 20.15 \\
$3 \times 3$ & 5 & $10^{1000}$ & 19.72 \\
$3 \times 3$ & 7 & $10^{1000}$ & 19.74 \\
\hline
\end{tabular}

our symmetric group. Once we have multiplied $M$ by itself a few times we get group ring elements of random length mixing throughout the matrix entries.

Random group ring elements from $\mathbb{Z}_{2}\left[S_{5}\right]$ have coefficients either 0 or 1 for each of the 120 elements of $S_{5}$. A simple binomial distribution calculation shows that with probability around $93 \%$ a random element of this group ring has a total number of nonzero terms between 50 and 70.

5.1. Experimental results on the Decision Diffie-Hellman assumption. We should note that for those experiments that were carried out using $2 \times 2$ matrices, it is reasonable to assume that if the results hold in the smaller matrix size, they will also hold for $3 \times 3$ matrices. In order to test the DDH assumption we need to look at the two distributions: one generated by $\left(M^{a}, M^{b}, M^{a b}\right)$ and the other generated by $\left(M^{a}, M^{b}, M^{c}\right)$ for a random $c$. Ideally, we would like the two distributions to be indistinguishable.

To verify that, we have run the following 3 experiments. In the first experiment, we verify that, as the common sense suggests, $M^{a b}$ has the same distribution as $M^{c}$. In the second experiment, we verify that $M^{a}$ is distributed "uniformly", i.e., like a randomly selected matrix $N$. A "randomly selected" matrix here means a matrix whose entries are random elements of the platform group ring. In turn, a random element of the group ring is selected by selecting each coefficient uniformly randomly from the ring of coefficients (in our case, from $\mathbb{Z}_{7}$ ). 
Combining the results of these two experiments, we see that each component in the triple $\left(M^{a}, M^{b}, M^{a b}\right)$ is uniformly distributed (for random $\left.a, b\right)$ in the sense described above. Now our final experiment verifies that the whole triple $\left(M^{a}, M^{b}, M^{a b}\right)$ is distributed like a triple of independently selected random matrices $\left(N_{1}, N_{2}, N_{3}\right)$, and therefore the distribution is indistinguishable from that of $\left(M^{a}, M^{b}, M^{c}\right)$ since the latter, too, is distributed like a triple of independently selected random matrices according to the previous experiments.

A more detailed description of the three experiments is below.

In the first experiment, we picked $a$ and $b$ randomly from the interval $\left[10^{22}, 10^{28}\right]$, and $c$ randomly from $\left[10^{44}, 10^{55}\right.$, so that $c$ had about the same size as the product $a b$. To get a clearer picture of how different or similar these final matrices were, we looked at each entry of the matrix. For each choice of a random matrix $M$ and random $a, b$, and $c$ we computed the matrices $M^{a b}$ and $M^{c}$. This was repeated 500 times and we created a table that was updated after each run with the distribution of elements of $S_{5}$ for each entry of the matrix. We were working with $M_{2}\left(\mathbb{Z}_{7}\left[S_{5}\right]\right)$.

After 500 runs we created Q-Q plots of entries of $M^{a b}$ versus entries of $M^{c}$, where we use the notation $M=\left(\begin{array}{ll}a_{1} & a_{2} \\ a_{3} & a_{4}\end{array}\right)$. Q-Q plots (or quantile plots) are a graphical method of comparing the quantiles of the cumulative distribution function (cdf) $F$ versus the corresponding quantiles of the cdf $G$. The functions are parameterized by $p$, where $p \in[0,1]$. One axis represents $F^{-1}(p)$ and the other axis represents $G^{-1}(p)$. If the two cdf's are identical, then the Q-Q plot will be that of $y=x$. It will also be a straight line if the distributions are of the same type, but have different mean and standard deviation, see [3] for more details.

As can be seen from Figure 1, it appears that the distributions of each of the matrices $M^{a b}$ and $M^{c}$ are indeed identical, which experimentally confirms what the common sense suggests.

In the second experiment, we verify that $M^{a}$ is distributed "uniformly", i.e., like a randomly selected matrix $N$. We also verify thereby that no information is leaked about $a$ by publishing $M^{a}$, for a given $M$. The experimental setup was similar to the previous one, only here we chose two random matrices $M$ and $N$, and a random integer $a \in\left[10^{44}, 10^{55}\right]$. Again we produced a Q-Q plot for the two distributions, see Figure 2. From the plot, it is clear that $M^{a}$ is indistinguishable from a random matrix $N$.

Finally, we ran a third experiment to ensure the independence of matrix entries from one another in the triple $\left(M^{a}, M^{b}, M^{a b}\right)$ by comparing its distribution to that of the triple of independently selected random matrices $\left(N_{1}, N_{2}, N_{3}\right)$. This is a valid and important question to ask as the information contained within the first two elements of the triple, which were shown to be random previously, may affect $M^{a b}$ in a predictable way. To this end, we ran 30,000 experiments four times, where for each element of $S_{5}$ we counted the frequency of coefficients of $\mathbb{Z}_{7}$ that occurred in the entries of each of the matrices in $\left(M^{a}, M^{b}, M^{a b}\right)$. We used the same $M$ in each experiment, but varied $a$ and $b$.

More specifically, we formed triples (one entry for each entry of the triple of matrices) consisting of the concatenation of the coefficients in the respective entry of the matrices for the same element of $S_{5}$. For example, if the coefficient at the same element of $S_{5}$ in the upper left corner entry of the first matrix is 0 , in the second matrix it is 5 , and in the third matrix it is 1 , then the concatenated coefficient is 051 . Thus, there is a total of $7^{3}=343$ concatenated coefficients. 
$Q Q$ Plot of frequency of elements of $S_{5}$ occuring in entries of $M^{\mathrm{ab}} v \mathrm{M}^{\mathrm{c}}$
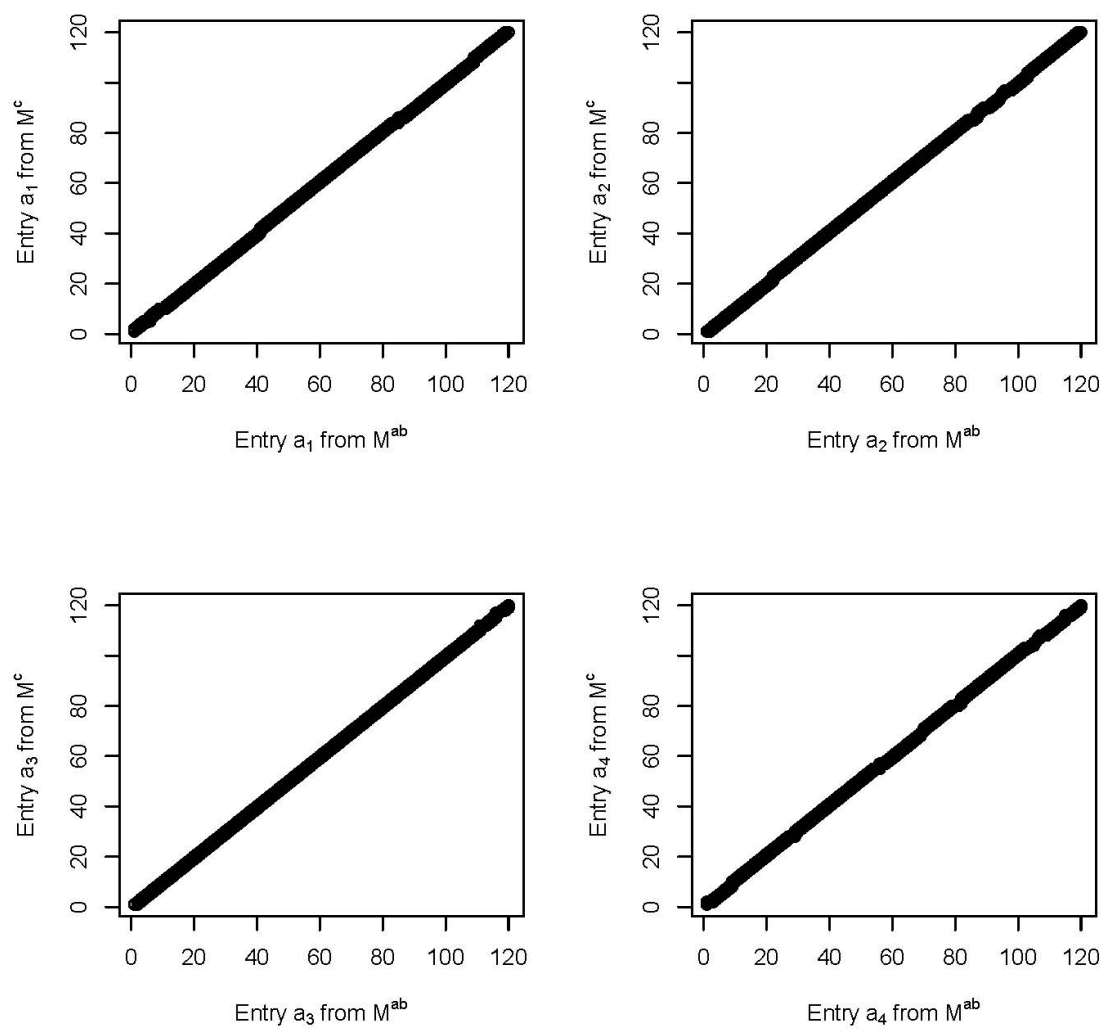

Figure 1. DDH results for $M^{a b}$ vs. $M^{c}$

We counted the occurrence of such triples throughout the experiments for random choices of $a$ and $b$ in the same range as in the previous experiments. We hypothesized that these coefficient triples would be uniformly distributed over $\mathbb{Z}_{7}^{3}$, each occurring with probability $1 / 7^{3}$. Since we performed 30,000 such experiments (four times), we anticipated that each element of this distribution would show up approximately $30,000 / 7^{3} \sim 87$ times.

We reproduced a section of these results in the Table 2, where we only used a portion of the table for the $a_{11}$ entry of the matrices because of the space constraints. Results for other entries are similar. The columns represent elements of $S_{5}$ (i.e., in the full table there would be 120 columns), the rows represent concatenated coefficients of the triples from $\mathbb{Z}_{7}^{3}$ (i.e., in the full table there would be $7^{3}=343$ rows), and the values in the table show the frequency of occurrence of the coefficients. All tables have the same "random" structure, and it can be seen that there appears to be no particular skew in the expected uniformity of the distribution of these coefficients, which allows us to conclude that the distribution of triples of all respective coefficients in $\left(M^{a}, M^{b}, M^{a b}\right)$ is, indeed, uniform on $\mathbb{Z}_{7}^{3}$. Since each component in the triple is itself uniformity distributed (as evidenced by our first two experiments), it follows that $M^{a b}$ is distributed independently of $\left(M^{a}, M^{b}\right)$. 
QQ Plot of frequency of elements of $S_{5}$ occuring in entries of $N$ vs $M^{a}$
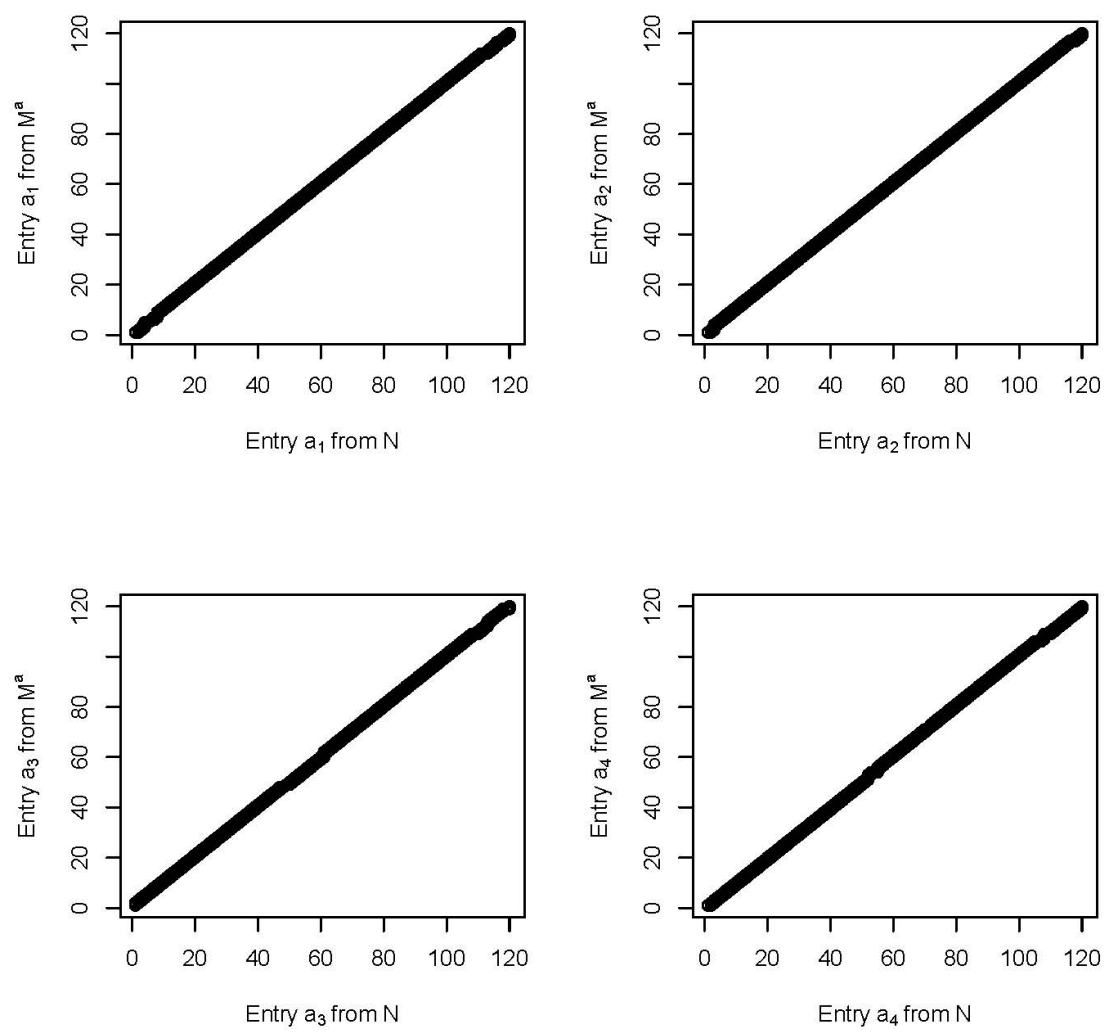

Figure 2. DDH results for $N$ vs. $M^{a}$

Table 2: Distribution of coefficient triples

\begin{tabular}{c|cccccccccccccccccc} 
& $s_{1}$ & $s_{2}$ & $s_{3}$ & $s_{4}$ & $s_{5}$ & $s_{6}$ & $s_{7}$ & $s_{8}$ & $s_{9}$ & $s_{10}$ & $s_{11}$ & $s_{12}$ & $s_{13}$ & $s_{14}$ & $s_{15}$ & $s_{16}$ & $s_{17}$ & $s_{18}$ \\
\hline$e_{1}$ & 87 & 90 & 83 & 90 & 86 & 93 & 85 & 84 & 88 & 88 & 93 & 88 & 85 & 77 & 88 & 94 & 93 & 91 \\
$e_{2}$ & 79 & 78 & 90 & 89 & 92 & 74 & 87 & 88 & 87 & 86 & 95 & 93 & 84 & 88 & 92 & 89 & 90 & 87 \\
$e_{3}$ & 86 & 86 & 83 & 89 & 95 & 91 & 93 & 90 & 94 & 85 & 82 & 87 & 84 & 84 & 86 & 84 & 89 & 89 \\
$e_{4}$ & 94 & 83 & 87 & 91 & 86 & 91 & 86 & 84 & 89 & 94 & 87 & 88 & 87 & 89 & 90 & 89 & 88 & 84 \\
$e_{5}$ & 81 & 88 & 82 & 85 & 85 & 94 & 86 & 89 & 92 & 84 & 94 & 90 & 93 & 86 & 83 & 79 & 93 & 85 \\
$e_{6}$ & 87 & 81 & 92 & 84 & 85 & 89 & 93 & 83 & 79 & 80 & 95 & 95 & 86 & 83 & 93 & 89 & 88 & 87 \\
$e_{7}$ & 87 & 84 & 82 & 91 & 96 & 88 & 88 & 81 & 97 & 89 & 88 & 86 & 90 & 90 & 93 & 85 & 96 & 88 \\
$e_{8}$ & 79 & 89 & 89 & 83 & 92 & 87 & 88 & 83 & 92 & 91 & 82 & 90 & 86 & 88 & 89 & 89 & 91 & 87 \\
$e_{9}$ & 82 & 79 & 83 & 86 & 88 & 81 & 90 & 93 & 88 & 89 & 87 & 85 & 88 & 91 & 85 & 90 & 87 & 92 \\
$e_{10}$ & 79 & 90 & 85 & 81 & 84 & 84 & 84 & 91 & 87 & 90 & 75 & 88 & 95 & 90 & 80 & 87 & 90 & 90 \\
$e_{11}$ & 90 & 80 & 96 & 90 & 78 & 89 & 86 & 87 & 91 & 83 & 90 & 88 & 93 & 94 & 92 & 85 & 80 & 90 \\
$e_{12}$ & 89 & 91 & 93 & 86 & 86 & 90 & 93 & 94 & 91 & 94 & 87 & 87 & 89 & 85 & 85 & 87 & 82 & 79 \\
$e_{13}$ & 90 & 81 & 90 & 87 & 88 & 89 & 89 & 83 & 85 & 87 & 86 & 92 & 93 & 87 & 94 & 81 & 94 & 90 \\
$e_{14}$ & 84 & 88 & 89 & 86 & 89 & 98 & 90 & 89 & 88 & 81 & 88 & 85 & 84 & 87 & 82 & 91 & 89 & 90 \\
$e_{15}$ & 86 & 86 & 86 & 87 & 94 & 95 & 90 & 88 & 85 & 84 & 86 & 83 & 87 & 90 & 92 & 92 & 88 & 88
\end{tabular}




\begin{tabular}{|c|c|c|c|c|c|c|c|c|c|c|c|c|c|c|c|c|c|c|}
\hline & $s_{1}$ & $s_{2}$ & $s_{3}$ & $s_{4}$ & $s_{5}$ & $s_{6}$ & $s_{7}$ & $s_{8}$ & $s_{9}$ & $s_{10}$ & $s_{11}$ & $s_{12}$ & $s_{13}$ & $s_{14}$ & $s_{15}$ & $s_{16}$ & $s_{17}$ & $s_{18}$ \\
\hline$e_{16}$ & 86 & 87 & 80 & 81 & 81 & 95 & 88 & 86 & 84 & 88 & 91 & 95 & 92 & 82 & 86 & 89 & 87 & 83 \\
\hline$e_{17}$ & 80 & 87 & 86 & 87 & 91 & 80 & 94 & 87 & 86 & 97 & 82 & 85 & 85 & 91 & 91 & 89 & 93 & 89 \\
\hline$e_{18}$ & 84 & 89 & 82 & 89 & 91 & 89 & 88 & 92 & 81 & 82 & 92 & 88 & 82 & 87 & 88 & 84 & 87 & 81 \\
\hline$e_{19}$ & 78 & 88 & 85 & 83 & 92 & 84 & 86 & 97 & 86 & 89 & 87 & 87 & 80 & 87 & 92 & 87 & 94 & 88 \\
\hline$e_{20}$ & 91 & 95 & 85 & 89 & 94 & 86 & 96 & 88 & 88 & 91 & 82 & 89 & 78 & 90 & 88 & 89 & 89 & 87 \\
\hline$e_{21}$ & 85 & 89 & 87 & 82 & 88 & 85 & 89 & 94 & 79 & 81 & 86 & & 0 & 86 & 39 & 86 & 90 & 81 \\
\hline$e_{22}$ & 85 & 92 & 86 & 83 & 87 & 85 & 84 & 78 & 81 & 85 & 83 & & 92 & 95 & 93 & 90 & 90 & 87 \\
\hline$e_{23}$ & 84 & 91 & 86 & 86 & 83 & 8 & 84 & 89 & 38 & 2 & 5 & & 87 & 90 & 84 & 79 & 82 & 81 \\
\hline$e_{24}$ & 97 & 83 & 93 & 93 & 90 & 9 & 88 & 95 & 86 & 81 & 88 & & 83 & 88 & 86 & 99 & 94 & 85 \\
\hline$e_{25}$ & 88 & 83 & 92 & 88 & 85 & 82 & 90 & 82 & 88 & 86 & 92 & 8 & 86 & 86 & 87 & 83 & 84 & 88 \\
\hline$e_{26}$ & 86 & 89 & 78 & 85 & 93 & 8 & 8 & 85 & 84 & 87 & 87 & & 102 & 86 & 93 & 91 & 91 & 92 \\
\hline$e_{27}$ & 90 & 83 & 77 & 81 & 94 & 85 & 86 & 83 & 90 & 86 & 87 & 9 & 90 & 82 & 79 & 95 & 83 & 85 \\
\hline$e_{28}$ & 85 & 79 & 86 & 83 & 80 & 8 & 8 & 88 & 85 & 86 & 92 & & 88 & 87 & 84 & & & 91 \\
\hline$e_{29}$ & 91 & 94 & 86 & 92 & 88 & 82 & 93 & 85 & 88 & 93 & 88 & & 85 & 92 & 77 & & & 88 \\
\hline$e_{30}$ & 97 & 91 & 88 & 87 & 88 & 88 & 8 & 87 & 89 & 89 & 82 & & 82 & 94 & 84 & 87 & & 91 \\
\hline$e_{31}$ & 89 & 91 & 92 & 87 & 97 & 88 & 89 & 83 & 89 & 92 & 84 & & 78 & 89 & 81 & 101 & & 86 \\
\hline$e_{32}$ & 88 & 84 & 81 & 90 & 80 & 91 & 90 & 89 & 89 & 8 & 89 & & 93 & 91 & 100 & 8 & 88 & 87 \\
\hline$e_{33}$ & 82 & 90 & 81 & 86 & 94 & 93 & 93 & 91 & 88 & 88 & 85 & 8 & 79 & 92 & 82 & 8 & 84 & 87 \\
\hline$e_{34}$ & 87 & 91 & 91 & 92 & 86 & 85 & 94 & 85 & 79 & 94 & 82 & 80 & 87 & 89 & 89 & 81 & 93 & 90 \\
\hline$e_{35}$ & 85 & 90 & 88 & 83 & 88 & 82 & 90 & 92 & 88 & 88 & 90 & 91 & 77 & 90 & 91 & 9 & 87 & 91 \\
\hline$e_{36}$ & 79 & 90 & 89 & 86 & 95 & 90 & 89 & 87 & 90 & 8 & 93 & 9 & 8 & 84 & 80 & 9 & 93 & 84 \\
\hline$e_{37}$ & 90 & 81 & 82 & 97 & 87 & 92 & 89 & 81 & 80 & 88 & 91 & 9 & 9 & 90 & 86 & 8 & $8:$ & 96 \\
\hline$e_{38}$ & 95 & 91 & 91 & 86 & 79 & 91 & 93 & 83 & 82 & 87 & 86 & 9 & 8 & 83 & 9 & 9 & 85 & 85 \\
\hline$e_{39}$ & 86 & 87 & 90 & 84 & 96 & 80 & 89 & 82 & 90 & 80 & 91 & 8 & 80 & 79 & 82 & & 98 & 91 \\
\hline$e_{40}$ & 92 & 87 & 92 & 80 & 84 & 91 & 90 & 88 & 91 & 9 & 86 & 8 & 86 & 92 & 86 & 9 & 92 & 87 \\
\hline$e_{41}$ & 84 & 90 & 90 & 91 & 83 & 86 & 91 & 90 & 88 & 84 & 88 & 86 & 89 & 82 & 83 & 9 & 92 & 90 \\
\hline$e_{42}$ & 90 & 90 & 86 & 76 & 96 & 86 & 87 & 80 & 89 & $8:$ & 87 & 9 & $8 \varepsilon$ & 89 & 84 & 9 & 89 & 86 \\
\hline$e_{43}$ & 81 & 86 & 97 & 83 & 89 & 84 & 88 & 88 & 83 & 84 & 96 & 87 & 87 & 90 & 91 & 8 & 91 & 87 \\
\hline$e_{44}$ & 86 & 82 & 90 & 89 & 76 & 87 & 93 & 81 & 83 & 9 & 85 & 8 & 90 & 86 & 90 & 9 & 84 & 90 \\
\hline$e_{45}$ & 88 & 95 & 88 & 88 & 95 & 91 & 83 & 92 & 92 & 86 & 82 & 8 & 94 & 87 & 88 & 92 & 83 & 90 \\
\hline$e_{46}$ & 93 & 87 & 96 & 80 & 89 & 90 & 86 & 84 & 87 & 100 & 85 & 9 & 89 & 93 & 96 & 84 & 91 & 85 \\
\hline$e_{47}$ & 92 & 85 & 85 & 85 & 91 & 91 & 87 & 88 & 83 & 84 & 87 & 8 & 89 & 83 & 89 & 86 & 84 & 83 \\
\hline$e_{48}$ & 90 & 87 & 82 & 99 & 76 & 82 & 84 & 82 & 83 & 9 & 83 & 94 & 92 & 87 & 93 & 86 & 86 & 82 \\
\hline$e_{49}$ & 93 & 82 & 85 & 86 & 85 & 87 & 91 & 85 & 80 & 91 & 94 & 87 & 92 & 90 & 90 & 87 & 86 & 96 \\
\hline$e_{50}$ & 90 & 78 & 85 & 83 & 85 & 88 & 93 & 82 & 84 & 87 & 92 & 82 & 84 & 89 & 85 & 81 & 84 & 88 \\
\hline$e_{51}$ & 90 & 90 & 83 & 86 & 97 & 87 & 88 & 90 & 90 & 92 & 88 & 8 & 96 & 86 & 90 & 90 & 88 & 98 \\
\hline$e_{52}$ & 88 & 82 & 92 & 92 & 88 & 83 & 94 & 92 & 91 & 92 & 89 & 89 & 87 & 91 & 81 & 81 & 87 & 88 \\
\hline$e_{53}$ & 88 & 89 & 89 & 86 & 92 & 86 & 85 & 86 & 90 & 93 & 75 & 90 & 91 & 95 & 87 & 84 & 92 & 83 \\
\hline$e_{54}$ & 89 & 91 & 88 & 92 & 82 & 84 & 95 & 84 & 82 & 82 & 85 & 86 & 91 & 93 & 93 & 96 & 84 & 75 \\
\hline$e_{55}$ & 88 & 88 & 84 & 88 & 82 & 94 & 100 & 89 & 84 & 88 & 79 & 89 & 90 & 85 & 88 & 83 & 85 & 85 \\
\hline
\end{tabular}

5.2. Experimental results on low orbits. Here we address the following "low orbits" question: we want to make sure that powers of the public matrix $M$ in our semigroup do not end up in an orbit of low order. This means that if Alice chooses a random integer $a$, we cannot have $M^{n}=M^{k}$, for $n<k<<a$ (similarly for $b$ chosen by Bob). If this were the 
case, then the eavesdropper Eve could first determine $n$ and $k$, then she could find the values of $c$ and $d$, where $1 \leq c, d \leq k$, such that $M^{a}=M^{c}$ and $M^{b}=M^{d}$. The shared secret key then could be computed as

$$
M^{a b}=\left(M^{a}\right)^{b}=\left(M^{c}\right)^{b}=\left(M^{b}\right)^{c}=\left(M^{d}\right)^{c}=M^{c d} .
$$

This is similar to the problem of finding a generator (i.e., an element of maximum order) in the multiplicative group of $\mathbb{Z}_{p}$, the original platform for the Diffie-Hellman protocol. Since we are dealing with a semigroup (of matrices) where most elements are not invertible and therefore do not have an "order" in the usual sense, we consider those orbits instead.

While it is conceivable that for a random matrix from $\mathbb{Z}_{7}\left[S_{5}\right]$ the length of such an orbit is going to be huge, we realize that when we are providing Alice and Bob with a matrix $M$, we have to at least have some solid lower bound for the length of an orbit for powers of $M$. Here is one possible approach.

The matrix $M$ will be a product of two matrices: $M=M_{1} \cdot S$, where $M_{1}$ is a random invertible matrix from $\mathbb{Z}_{7}\left[S_{5}\right]$, and $S$ is a "scalar" matrix that has zeros off the diagonal and each element on the diagonal is $s=\left(3+g_{1}\right)\left(3+g_{2}\right)\left(3+g_{3}\right)\left(3+g_{4}\right)\left(3+g_{5}\right)\left(3+g_{6}\right)(5+h)$. Here $g_{i}$ are elements of $S_{5}$ that generate different subgroups of order 5 , and $h$ is a product of a 2 -cycle and a 3 -cycle. The element $s$ is not invertible because it is a zero divisor. To see this, write $(5+h)$ as $(h-2)$ and multiply it by $\sum_{i+j=5} h^{i} 2^{j}$ to get $\left(h^{6}-2^{6}\right)=0$ since $h^{6}=2^{6}=1$ in our group ring. Therefore, the matrix $S$ is not invertible either. We have run a computer program trying to detect an orbit generated by powers of $S$. While our program has not terminated in the allotted time (several weeks), we know that there are no orbits up to $s^{10^{10}}$. Then, for a random invertible matrix $M_{1}$, we have just computed powers of $M_{1}$ up to $M_{1}^{10^{10}}$, and none of these powers was the identity matrix (or even a diagonal matrix). We note that looking for orbits going through powers of a non-invertible matrix $M$ would consume much more resources and was, in fact, infeasible beyond $M^{10^{6}}$ given our computational resources. This is because once each power of $M$ is computed, it needs to be stored and eventually compared to all other powers of $M$. For an invertible matrix $M_{1}$, on the other hand, we do not need to store any powers to find its order.

Now we claim that with overwhelming probability, if we have a random invertible matrix $M_{1}$ with the property that the powers of $M_{1}$ up to $10^{10}$ are not diagonal matrices, then the powers of $M_{1} \cdot S$ up to $10^{10}$ do not have any orbits. To see this, let us assume that the matrices $M_{1}$ and $S$ commute; if our claim is valid under this assumption then it is also valid without this assumption since adding a relation $M_{1} S=S M_{1}$ is like considering a homomorphic image: equalities will be preserved.

Suppose now that we have $\left(M_{1} S\right)^{n}=\left(M_{1} S\right)^{n+k}$ for some positive integers $n, k$, with $k<10^{10}$. If $M_{1}$ and $S$ commute, this yields $M_{1}^{n} S^{n}=M_{1}^{n+k} S^{n+k}$. Since $M_{1}$ is invertible, we can cancel $M_{1}^{n}$ and get $S^{n}=M_{1}^{k} S^{n+k}$, and then

$$
\left(M_{1}^{k} S^{k}-I\right) \cdot S^{n}=O,
$$

where $I$ is the identity matrix and $O$ is the zero matrix. While it is possible that the product of two nonzero matrices is the zero matrix, the probability of this to happen is negligible, given that the matrix $M_{1}^{k} S^{k}-I$ is not even diagonal (with overwhelming probability) if $k<10^{10}$, as our experiments suggest. The matrix $S^{n}$, on the other hand, is diagonal; therefore, for the 
displayed equality above to hold, every non-zero element $a_{i j}$ of the matrix $\left(M_{1}^{k} S^{k}-I\right)$ has to be a zero divisor such that $a_{i j} \cdot r=0$, where $r$ is the element on the diagonal of the matrix $S^{n}$ (the latter is obviously a scalar matrix). This (somewhat informal) argument shows that $k>10^{10}$ with overwhelming probability. We realize that this lower bound may not be very impressive, but more convincing lower bounds may be based on less convincing arguments. We believe that, in fact, $k>10^{80}$ with overwhelming probability, but at the time of this writing we do not have a convincing argument to support that belief.

To conclude this section, we say a few words about sampling invertible matrices. There are several techniques for doing this; here we give a brief exposition of one of them. We start with an already "somewhat random" matrix, for which it is easy to compute the inverse. An example of such a matrix is a lower/upper triangular matrix, with invertible elements on the diagonal:

$$
U=\left(\begin{array}{ccc}
g_{1} & u_{1} & u_{2} \\
0 & g_{2} & u_{3} \\
0 & 0 & g_{3}
\end{array}\right) .
$$

Here $g_{i}$ are random elements of the group $S_{5}$, and $u_{i}$ are random elements of the group ring $\mathbb{Z}_{7}\left[S_{5}\right]$. We then take a random product, with 20 factors, of such random invertible upper and lower triangular matrices, to get our invertible matrix $M_{1}$.

\section{6. "STANDARD" ATtACKS}

In this section, we discuss why three "standard" attacks on the "classical" discrete logarithm problem do not work with our platform semigroup.

6.1. Baby-step giant-step algorithm. One known method of attacking the "classical" discrete logarithm problem, due to Shanks [8], is the baby-step giant-step algorithm. The algorithm computes discrete logarithms in a group of order $q$ in $O(\sqrt{q} \operatorname{polylog}(q))$ time, where polylog $(q)$ is $O\left((\log (q))^{c}\right)$ for some constant $c$. If adapted to our situation, this algorithm would look as follows.

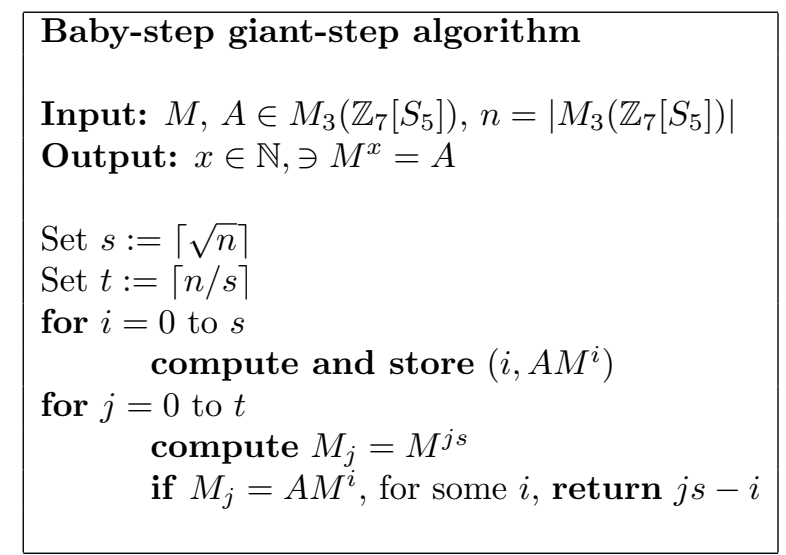

There are a couple of points that have to be made about this algorithm. The first is that we need to produce a good method of storing the matrices. This could be possible with a hash function, in which case insertion and lookup is constant in time. However, our matrices 
are fairly complex objects, and we need to take into account the storage requirements of the algorithm.

Furthermore, we should note that the order of our chosen random matrix $M$ is much smaller than that of the whole group ring. Hence, it may be possible to use a smaller value of $n$ as an input. However, this requires knowledge of the order of $M$. As little is known about the structure of this group ring, we are not guaranteed that the order exists in the usual sense. We are basically back to looking for orbit collisions as in our Section 5.2 .

Each entry in the matrix can be represented by a sequence of 120 (three-bit) coefficients. We can use a 360 bit string where we encode each three-bit sequence with the value of the coefficient of that polynomial term in $\mathbb{Z}_{7}\left[S_{5}\right]$. Hence each matrix will need $360 \times 4$ bits of storage. In this algorithm we are required to store $\sqrt{\left|M_{3}\left(\mathbb{Z}_{7}\left[S_{5}\right]\right)\right|}=\sqrt{7^{540}} \sim 10^{456}$ such matrices. In order to store all these matrices we would need $1440 \times 10^{456}$ bits of space. This works out to about $10^{446} \mathrm{~TB}$ of (memory or hard drive) space. Thus, it looks like this algorithm is infeasible already in terms of space. Of course, storing the arrays can be optimized, e.g. we do not need to store entries with zeroes. However, the amount of information that we need to store, $10^{456}$ matrices, is still too big even if we only store the number of non-zero terms in the polynomials.

One approach often suggested to decrease space requirements is to decrease $s$, hence increasing $t$. In this case the algorithm instead of running in $O(\sqrt{n})$ time will run in $O(n / t)$ time. Every time we reduce by half the storage requirements, we end up doubling the running time of the algorithm. However, regardless of what $s$ and $t$ are chosen to be we still need to perform $s+t$ group operations in the two loops. Given our constraints, the number of group operations is minimized when $s=t=\sqrt{n}$. Hence, we need at least $10^{457}$ group operations to run this algorithm, which is again computationally infeasible.

6.2. Other attacks. There are two other algorithms that have been suggested for solving the "classical" discrete logarithm problem. The first is the Pohlig-Hellman algorithm [6]. This algorithm relies on the order of a group element and the generalized Chinese remainder theorem to break the problem into smaller subproblems.

Specifically, suppose the order of the element $g \in G$ is $q$. In the Diffie-Hellman scheme we wish to find an $x$ such that $g^{x}=y$. Suppose we know a factorization

$$
q=\prod_{i=1}^{n} q_{i},
$$

where the $q_{i}$ are relatively prime. Then we have

$$
\left(g^{q / q_{i}}\right)^{x}=\left(g^{x}\right)^{q / q_{i}}=y^{q / q_{i}}, \text { for } i=1, \ldots, n .
$$

By the Chinese remainder theorem we can write

$$
\mathbb{Z}_{q} \cong \mathbb{Z}_{q_{1}} \times \cdots \times \mathbb{Z}_{q_{n}}
$$

and we are left to solve $n$ instances of the discrete logarithm problem in the smaller groups, i.e., defining $g_{i}=g^{q / q_{i}}$, we must find the solutions $\left\{x_{i}\right\}_{i=1}^{n}$ for which $g_{i}^{x_{i}}=y^{q / q_{i}}=g^{x}$.

However, in our situation the order of matrices in $M_{3}\left(\mathbb{Z}_{7}\left[S_{5}\right]\right)$ does not relate to the size of the whole ring $M_{3}\left(\mathbb{Z}_{7}\left[S_{5}\right]\right)$. Again, under multiplication this ring is a semigroup, not a group, and the proportion of invertible elements in this semigroup is very small. Additionally, the 
size of this ring is $7^{1080}$, so the Chinese remainder theorem does not really help in breaking this problem into smaller parts. If, however, there was a way to break the problem into smaller subproblems, we would still need to solve the discrete logarithm problem in our setting, which so far as we know can only be done via brute force.

The second algorithm proposed for solving the "classical" discrete logarithm problem is Pollard's rho algorithm [7]. The inputs are group elements $M$ and $N$, and the output is an integer $n$ such that $M^{n}=N$. The algorithm first looks for an orbit, which has the general form $M^{a} N^{b}=M^{c} N^{d}$, for $a, b, c$ and $d \in \mathbb{N}$. This is done by using Floyd's cycle-finding algorithm. As long as $b \neq d$, one can take the logarithm with base $M$ to determine $n$ :

$$
\begin{aligned}
M^{a} N^{b} & =M^{c} N^{d} \\
\Rightarrow a+b \log _{M} N & =c+d \log _{M} N \\
\Rightarrow \frac{a-c}{d-b} & =\log _{M} N \\
\Rightarrow M^{\frac{a-c}{d-b}} & =N
\end{aligned}
$$

However, in applying Floyd's cycle-finding algorithm in Pollard's rho attack, the knowledge of the order of the cyclic group generated by $M$ is essential. In our situation, not only is the order of $M$ unknown, but more importantly, since a random $M$ is not going to be invertible with overwhelming probability, order considerations are not applicable, and therefore neither is Pollard's rho attack, at least in its standard form.

6.3. Quantum Algorithm Attacks. It is well known that many cryptographic protocols are vulnerable to quantum algorithm attacks [9]. In particular, the Diffie-Hellman protocol can be attacked using Shor's algorithm. This algorithm basically recasts the discrete logarithm problem as a hidden subgroup problem (HSP) and uses the quantum algorithms developed for HSP to recover the exponent.

We believe that our protocal is secure against such attacks. The HSP relies on the existence of a function $f: G \rightarrow S$, for some set $S$, such that $f$ is constanct on cosets of the unknown subgroup $H \leq G$ and also takes on distinct values for each coset. For the discrete log we define $f: \mathbb{Z}_{N} \times \mathbb{Z}_{N} \rightarrow G$, such that $f(a, b)=g^{a} x^{b}$, where $a, b \in \mathbb{Z}_{N}, g, x \in G, g^{\alpha}=x$ and $|g|=N$. We can rewrite this as $f(a, b)=g^{a+b \cdot \log _{g} x}$, and hence $f$ is constant on the sets $L_{c}=\left\{(a, b) \mid a+b \log _{g} x=c\right\}$.

In this setup the hidden subgroup we are seeking is

$$
H=L_{0}=\left\{(0,0),\left(\log _{g} x,-1\right),\left(2 \log _{g} x,-2\right), \cdots,\left(N \log _{g} x,-N\right)\right\} .
$$

To be able to apply this algorithm one would need to know the order of a matrix. However, this is not known a priori and it is also the case that invertible matrices are sparse in our setup. Hence in our setup the function $f$ is ill-defined.

Furthermore, given a random non-invertible matrix it is unlikely that the function $f$ will be distinct on cosets of the subgroup $H$ or even constant on the different cosets. To see this assume $M$ is a non-invertible matrix, then powers of $M$ will either end up in an orbit or will eventually become the zero matrix. If we are in an orbit, assume for example that $M^{9}=M^{15}$ and the exponent we are seeking is $\alpha=12$. The subgroup we are trying to identify is $H=$ $\{(0,0),(12,-1),(24,-2),(36,-3), \cdots\}$. From the setup we note that $(36,-3) \sim(18,-3)$, but $(18,-3) \notin H$, for if it were then $(36,-3)-(18,-3)=(18,0) \in H$, which is a contradiction. On 
the other hand, assume some power of $M$ is the zero matrix, say $M^{20}=0$, and again $\alpha=12$. In this case $f$ is no longer constant on the subgroup $H$ as $0=f(24,-2) \neq f(12,-1)=I$.

\section{Conclusions}

Our contribution here is proposing the semigroup of matrices (of a small size, $2 \times 2$ or $3 \times 3$ ) over the group ring $\mathbb{Z}_{7}\left[S_{5}\right]$, with the usual matrix multiplication operation, as the platform for the Diffie-Hellman key exchange scheme. What we believe is the main advantage of our platform over the standard $\mathbb{Z}_{p}^{*}$ platform in the original Diffie-Hellman scheme is that the multiplication of matrices over $\mathbb{Z}_{7}\left[S_{5}\right]$ is very efficient. In particular, in our setup multiplying elements is faster than multiplying numbers in $\mathbb{Z}_{p}$ for a large $p$. This is due to the fact that one can pre-compute the multiplication table for the group $S_{5}$ (of order 120), so in order to multiply two elements of $\mathbb{Z}_{7}\left[S_{5}\right]$ there is no "actual" multiplication involved, but just re-arrangement of a bit string of length $3 \times 120$. Also, no reduction modulo a large $p$ is involved.

To verify the security of using such a semigroup of matrices as the platform, we have experimentally addressed the Decision Diffie-Hellman assumption (Section 5) and showed, by using Q-Q plots (or quantile plots) that after 500 runs of the experiment, two distributions, one generated by $M^{a b}$ and the other generated by $M^{c}$ for a random $c$, are indistinguishable, thereby experimentally confirming the DDH assumption for our platform. Furthermore, no information is leaked from $M^{a}$ by comparing it to a random matrix $N$.

From the security point of view, the advantages of our platform over $\mathbb{Z}_{p}$ also include the fact that neither "standard" attacks (baby-step giant-step, Pohlig-Hellman, Pollard's rho) nor quantum algorithm attacks work with our platform, as we showed in Section 6 .

\section{REFERENCES}

[1] D. Boneh, The Decision Diffie-Hellman Problem, ANTS 1998, pp. 48-63.

[2] W. Diffie and M. E. Hellman, New Directions in Cryptography, IEEE Transactions on Information Theory IT-22 1976, 644-654.

[3] J. D. Gibbons, S. Chraborti, Nonparametric Statistical Inference, CRC Press, 1992.

[4] E. Kasper, Fast Elliptic Curve Cryptography in OpenSSL, Financial Cryptography and Data Security, 2011.

[5] A. Menezes, P. van Oorschot, and S. Vanstone, Handbook of Applied Cryptography, CRC Press 1996.

[6] S. Pohlig and M. Hellman, An Improved Algorithm for Computing Logarithms over GF(p) and its Cryptographic Significance, IEEE Transactions on Information Theory IT-24, 1978, 106-110.

[7] J. Pollard, Monte Carlo methods for index computation mod p, Mathematics of Computation 32, 1978, 331-334.

[8] D. Shanks, Class number, a theory of factorization and genera, Analytic Number Theory, Proceedings of Symposia on Pure Mathematics, 20, American Mathematical Society, 1971, pp. 415-440.

[9] P. Shor, Algorithms for Quantum Computation: Discrete Logarithms and Factoring, Proc. 35th Annual Symposium on Foundations of Computer Science (1994). IEEE Comput. Soc. Press, pp. 124-134.

\section{Appendix: A CHALlEnge}

Here we present a challenge relevant to our Diffie-Hellman-like scheme: given explicit $3 \times 3$ matrices $M, M^{a}$, and $M^{b}$ over the group ring $\mathbb{Z}_{2}\left[S_{5}\right]$, recover the matrix $M^{a b}$. Note that our recommended platform ring is actually $\mathbb{Z}_{7}\left[S_{5}\right]$, but we believe that breaking our challenge is currently infeasible even for $\mathbb{Z}_{2}\left[S_{5}\right]$. 


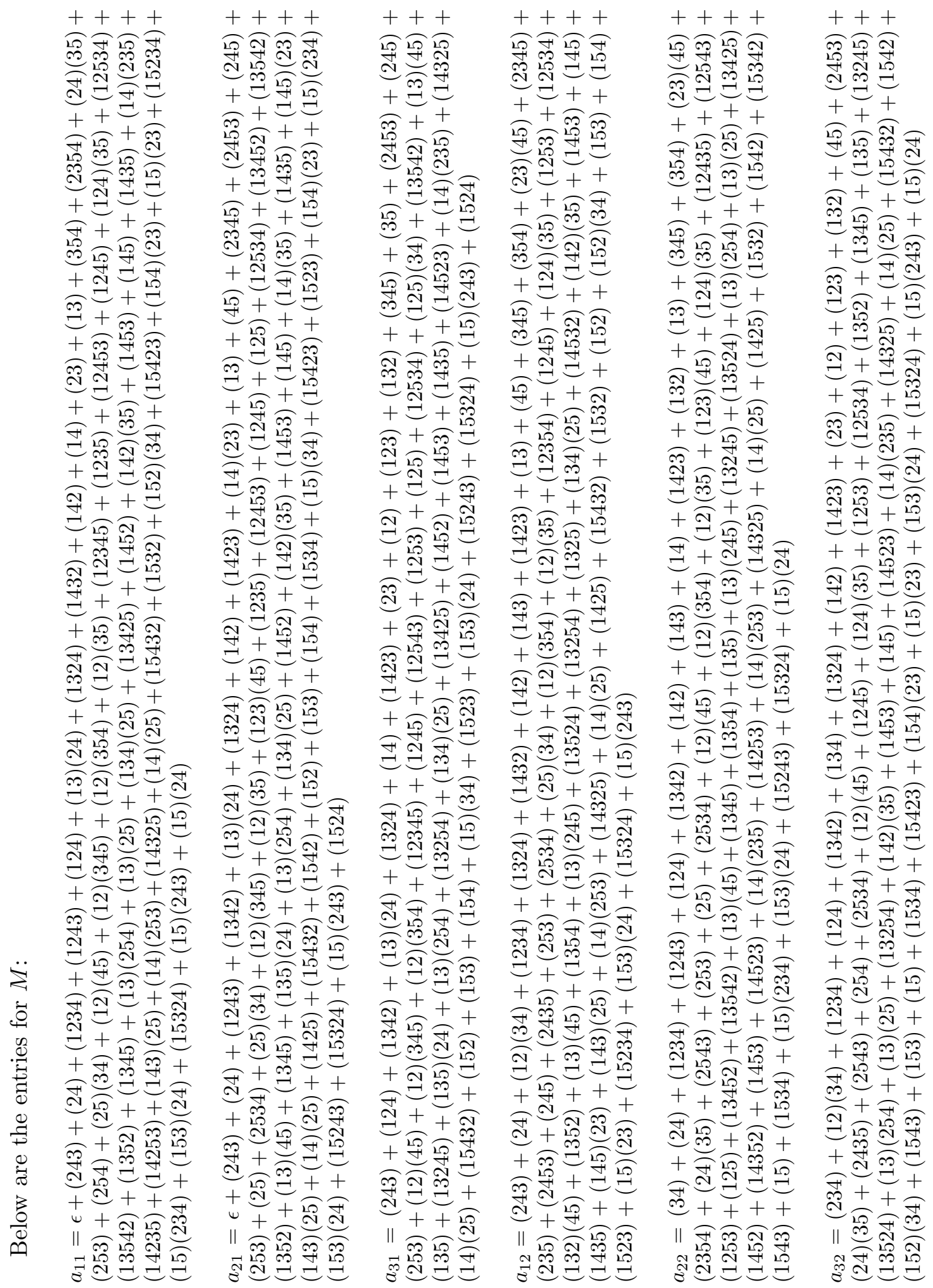



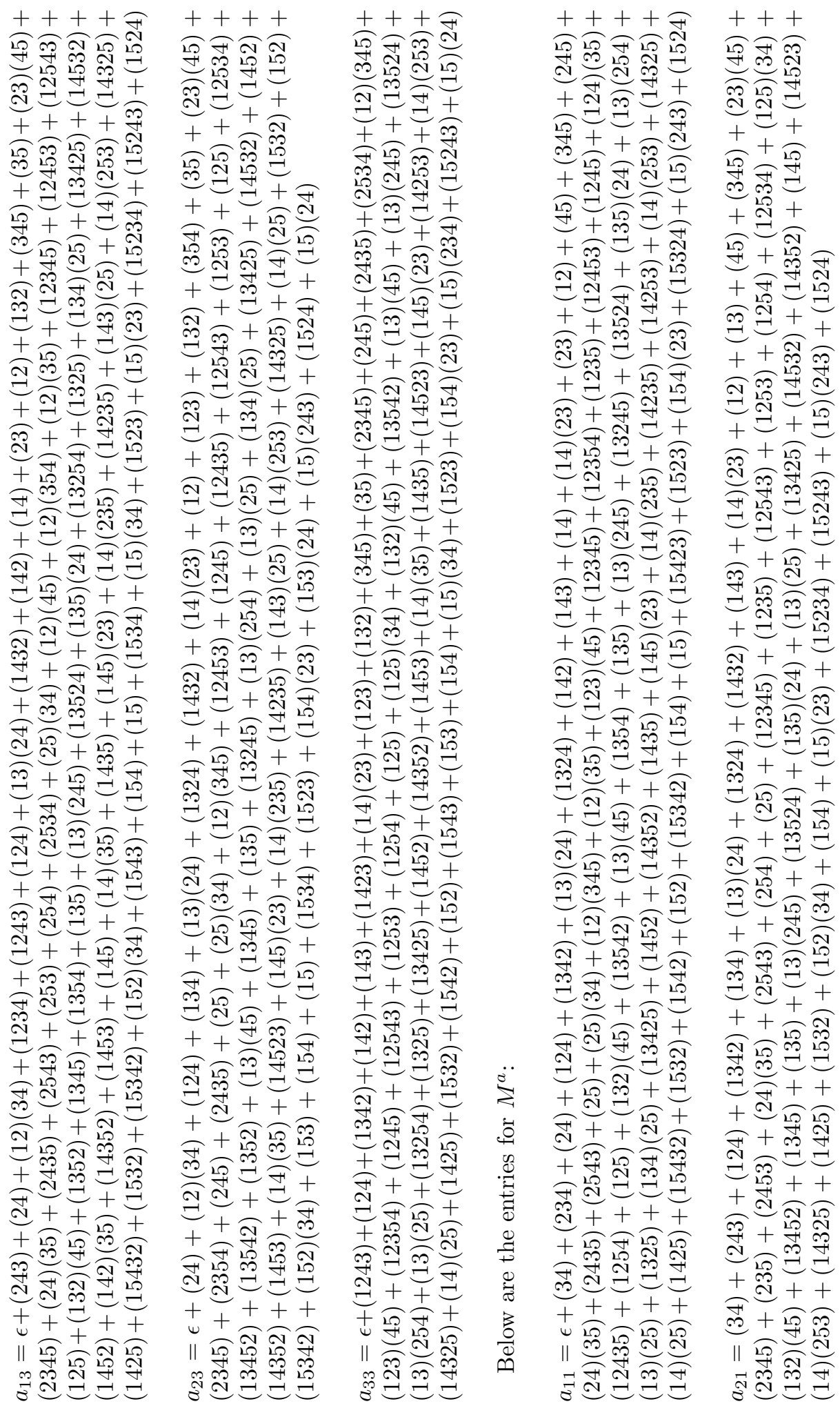

$+++$

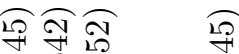

ป⿰⿻上丨⿶凵

$+\underset{+}{\beth}=+$

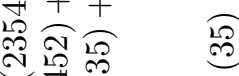

$+\stackrel{2}{\overparen{1}+}+$

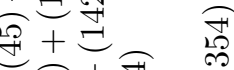

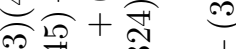

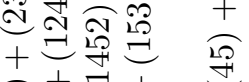

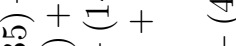

लำ $\overparen{2}$

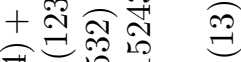

穴 + 寻

$+\overparen{2}+\overparen{2}$

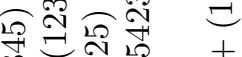

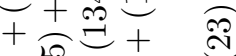

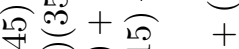

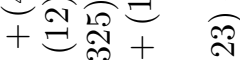

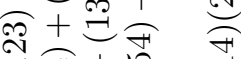

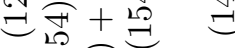

$+\stackrel{2}{*}++$

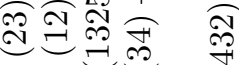

$++\underset{\overparen{C}}{ }$

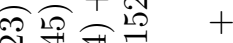

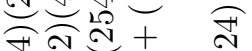

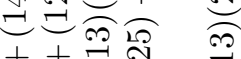

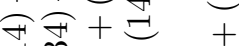

+ ㄴํㄴ?

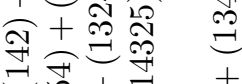

च

$+\overbrace{\overparen{2}}^{\circ}$

अิ

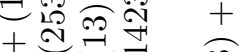

m+ $++\underset{7}{2}$

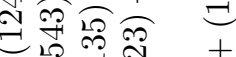

$+\mathrm{N}=$ i

क्ञ⿰冫欠 ॠ

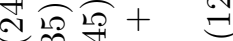

$+\stackrel{\sim}{\overparen{2}}+$

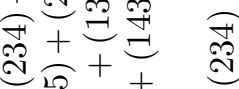

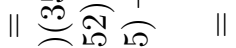

 

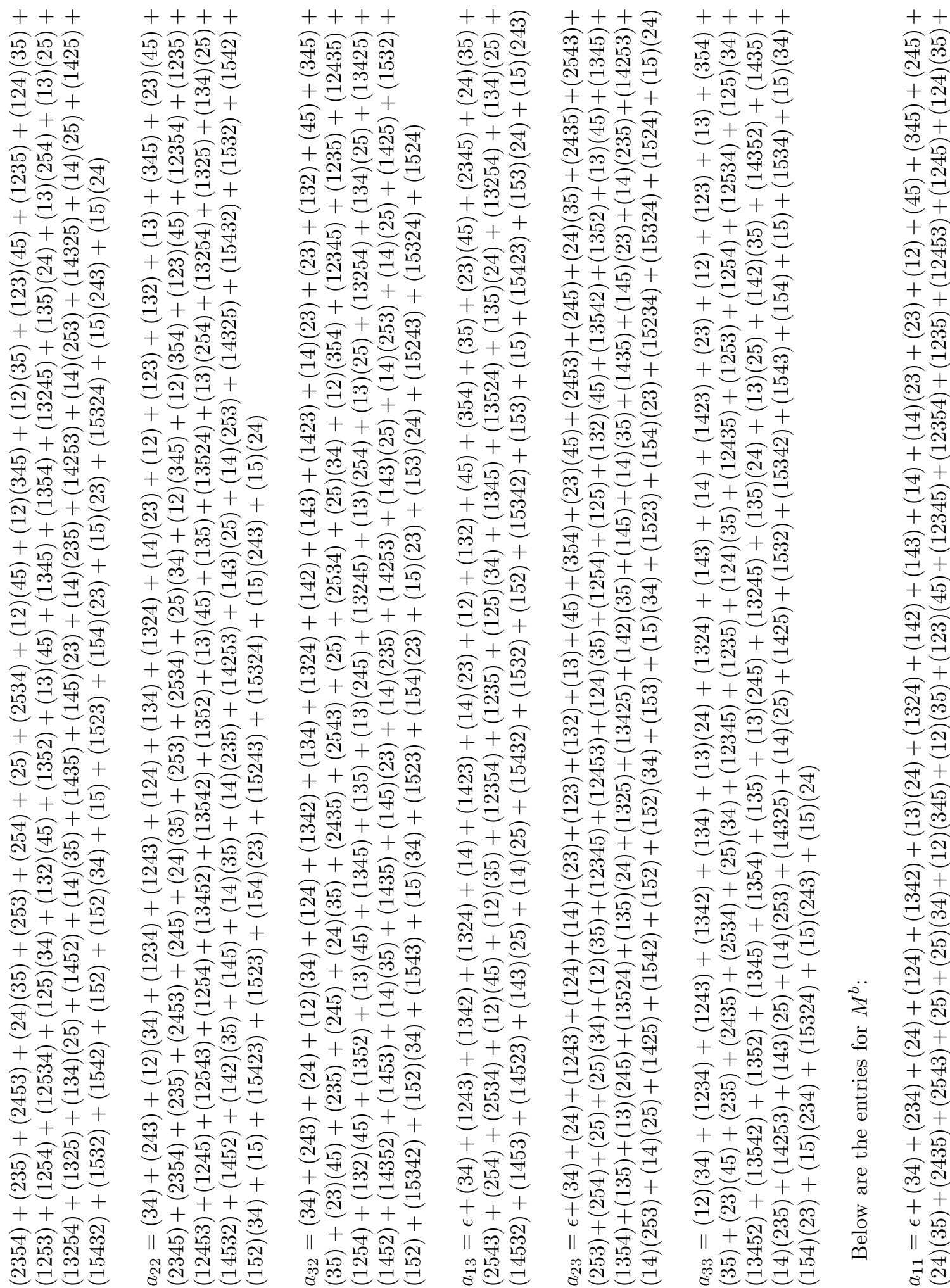

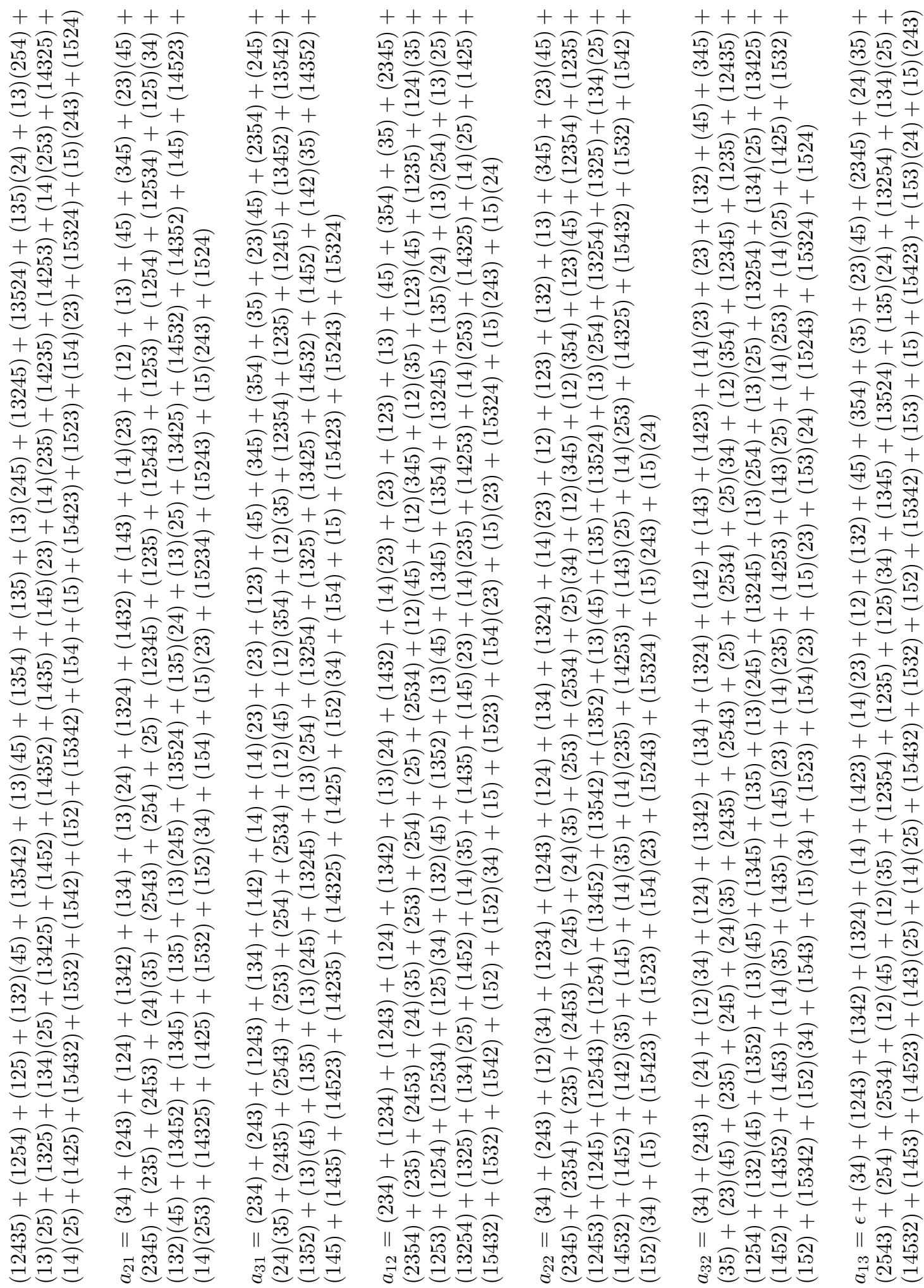


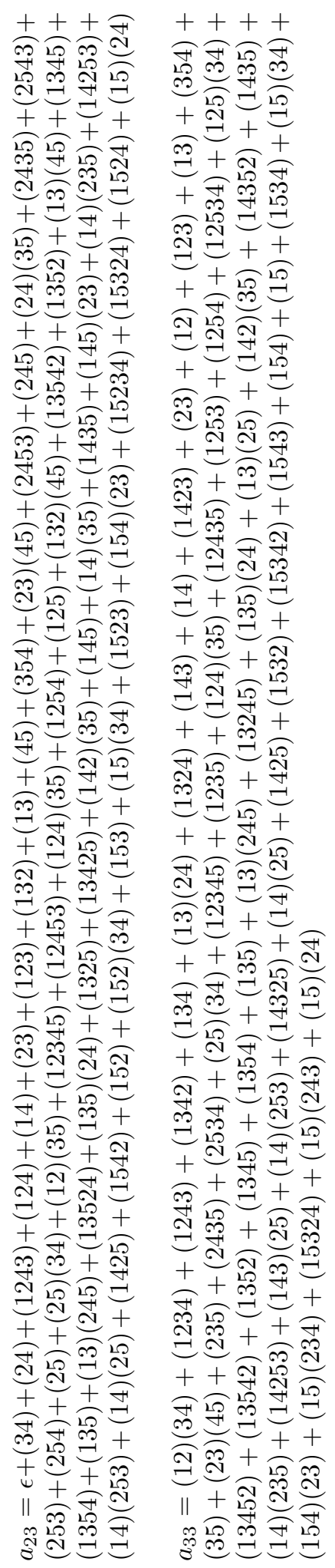


CUny Graduate Center and City Tech, City University of New York

E-mail address: DKahrobaei@GC.Cuny.edu

CUNy Graduate Center, City University of New York

E-mail address: ckoupparis@GC.Cuny.edu

The City College of New York and CUNy Graduate Center

E-mail address: shpil@groups.sci.ccny.cuny.edu 